\title{
Commentary: Genome-wide association study identifies 74 loci associated with educational attainment
}

\author{
Félix Hernández ${ }^{1,2 *}$ and Jesús Ávila ${ }^{1,2 *}$ \\ ${ }^{1}$ Centro de Biología Molecular Severo Ochoa (CSIC-UAM), Madrid, Spain, ${ }^{2}$ Centro de Investigación Biomédica en Red \\ Sobre Enfermedades Neurodegenerativas (CIBERNED, ISCIII), Madrid, Spain
}

Keywords: Alzheimer's disease, educational attainment, SNP, tau, tauopathies

\section{A commentary on}

Genome-wide association study identifies 74 loci associated with educational attainment by Okbay, A., Beauchamp, J. P., Fontana, M. A., Lee, J. J., Pers, T. H., Rietveld, C. A., et al. (2016). Nature 533, 539-542. doi: 10.1038/nature17671

\section{OPEN ACCESS}

Edited by:

Jean-Marc Taymans,

French Institute of Health and Medical

Research (Inserm), France

Reviewed by:

Emmanuel Planel,

Laval University, Canada

Alejandra Alonso,

College of Staten Island, USA

Dhaenens Claire-Marie,

University of Lille Nord de France,

France

*Correspondence:

Félix Hernández

fhernandez@cbm.csic.es

Jesús Ávila

javila@cbm.csic.es

Received: 29 September 2016 Accepted: 17 January 2017

Published: 31 January 2017

Citation:

Hernández F and Ávila J (2017) Commentary: Genome-wide

association study identifies 74 loci

associated with educational

attainment

Front. Mol. Neurosci. 10:23. doi: 10.3389/fnmol.2017.00023
It is generally assumed that social and other environmental factors like poverty are involved in educational attainment. However, a recently published genome-wide association study (GWAS) for educational attainment of 293,723 individuals (Okbay et al., 2016) identified 74 loci associated with the number of years of schooling completed, with eight of them showing the highest association. One of these genes showing a single-nucleotide polymorphism (SNP) linked to educational attainment was located on chromosome 17. The SNP was rs192818565 (dbSNP ID: has merged into rs62056842 T/G) in position chr17:45914149 (according to GRCh38.p7 assembly of human genome), located within the first intron on the MAPT gene (microtubule associated protein tau, Figure 1A; http://www.ncbi.nlm.nih.gov/projects/SNP/snp_ref.cgi?rs=62056842).

The human tau gene is located on chromosome 17: 45,894,382-46,028,334, and it contains 16 exons that by historical reasons have been numbered as shown in Figure 1A. Tau is mainly expressed in neurons, and by alternative splicing of exons 2, 3, and 10 yields different mRNA species in the central nervous system (Andreadis, 2005). Alternative splicing of exon 10 produces tau isoforms with either three (3R-tau, lacking exon 10) or four (4R-tau, including exon 10) tubulin/microtubule binding repeats. The locus where MAPT gene is present has been divided into two haplotypes, $\mathrm{H} 1$ and $\mathrm{H} 2$. Haplotype $\mathrm{H} 2$ is inverted with respect to $\mathrm{H} 1$. The inversion encompasses a number of genes (CRHR1, IMP5, MAPT, and NSF) and the absence of recombination between them has resulted in both haplotypes defined among other marks by 8 SNP (for a review see Caffrey and Wade-Martins, 2007). Non-inverted H1 haplotypes are more susceptibility to tauopathies and its promoter is more efficient at transcription level compared with H2 promoter (Kwok et al., 2004).

Tau protein is altered in many diseases, from Alzheimer's disease (AD) to Parkinson's disease (PD) and, as has been recently described, Huntington's disease (Avila et al., 2004; Fernández-Nogales et al., 2014; Iqbal et al., 2016). Tau missense mutations have been described in FTDP-17 while in other tauopathies tau levels or the 3R/4R-tau ratio are altered. While SNPs are roughly homogeneously distributed throughout the gene (Figure 1A, light blue boxes), clinical association studies have shown that SNPs labeled as "probably pathogenic" or "pathogenic" are mainly located at the $3^{\prime}$-end of the gene, where the coding exons are located (Figure 1A, purple boxes, http://www.ncbi.nlm.nih.gov/projects/SNP/). Conversely, the first intron where the SNP linked to educational attainment was located, has few of those. 


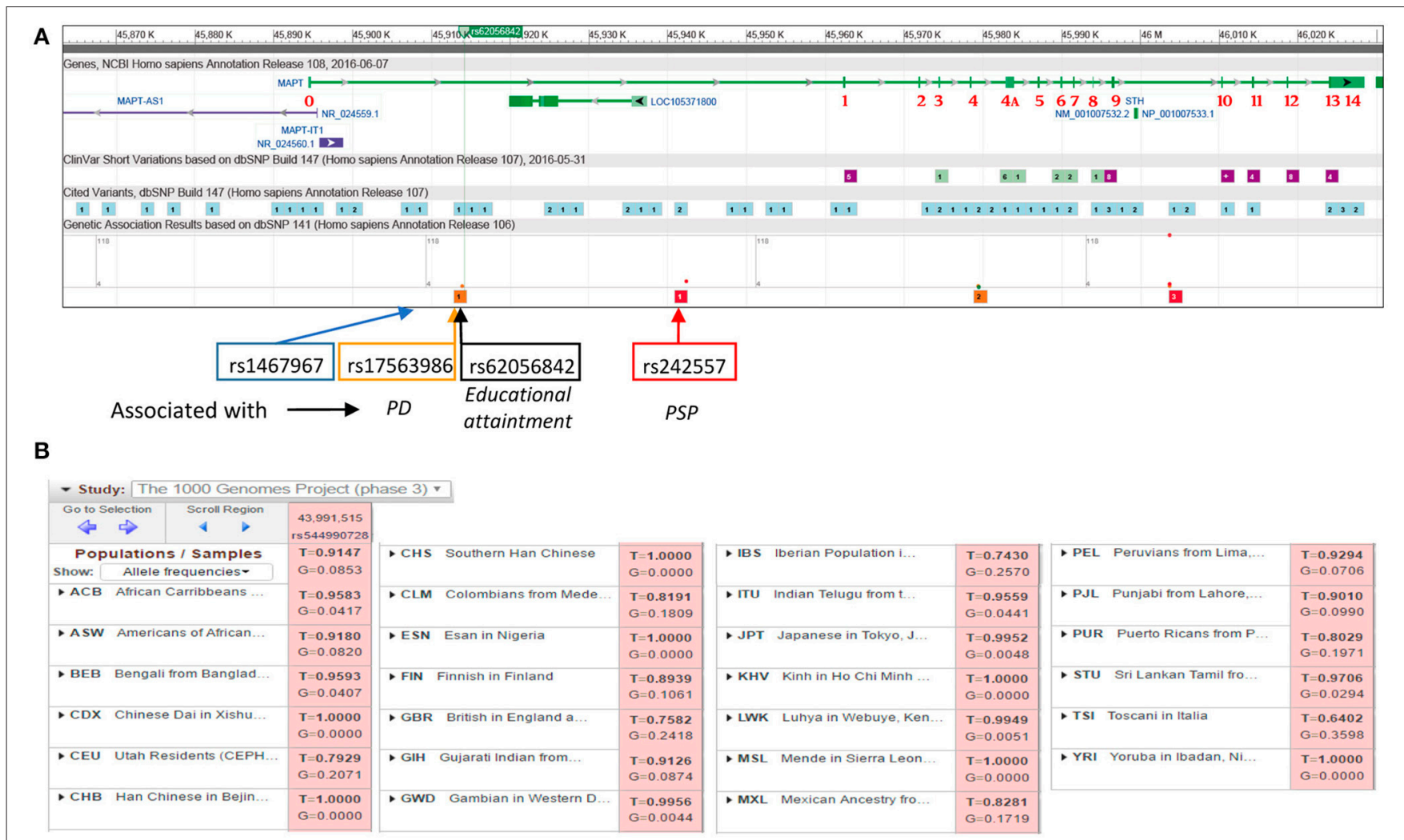

FIGURE 1 | MAPT gene. A single tau gene located on the human chromosome 17 is transcribed into the corresponding nuclear RNA that, by alternative splicing, yields several tau mRNAs. (A) Exons are labeled in red numbers. Sth indicates the existence of a DNA sequence encoding the protein saithoin within the intron between exons 9 and 10. ncRNAs MAPT-AS1, MAPT-IT1 and likely LOC105371800 are shown. Light blue boxes: SNP present in MAPT gene; purple boxes: SNP labeled as "probable pathogenic" or "pathogenic"; red and orange boxes: SNPs that genetic association studies have linked to a strong risk to major diseases (Parkinson disease -rs17563986- and progressive supranuclear palsy -rs242557-). Some SNPs used to describe MAPT haplotypes are flanking the SNP here commented on (rs62056842) and are located within that first intron ( $r$ 1467967 and rs242557). (B) Allele frequencies of SNP rs192818565 in diverse human populations (rs544990728 in 1000 genomes data base). Data and images have been taken from https://www.ncbi.nlm.nih.gov/variation/tools/1000genomes/.

Interestingly, genetic association studies have found associations between SNPs in the first intron (close to rs62056842; Figure 1A, red and orange boxes) and a strong associated risk to major diseases. Furthermore, two SNPs used to describe MAPT haplotypes are flanking the above mentioned SNP and are located within that first intron (rs1467967-position chr17:45908813- and rs242557 -position chr17:45942346-) (Myers et al., 2007).

Few studies have focused on intron 0 . As in many eukaryotic genes (Bradnam and Korf, 2008), the first intron is the longest one found in the MAPT gene likely because it harbors cis regulatory sequences (Chorev and Carmel, 2012). MAPT promoter presents two main characteristic: absence of TATA and CAAT boxes and a big G+C content (Gao et al., 2005). Thus, methylation of $\mathrm{CpG}$ islands present in exon and intron 0 can modulate tau expression likely altering accessibility to binding factors and transcription initiation factors (Caillet-Boudin et al., 2015). With these ideas in mind, it is tempting to speculate that rs62056842 SNP might affect that regulatory system. In fact CpG island close to rs242557 SNP is hypomethylated in PSP (Huin et al., 2016) and H1 haplotype increases risk for tauopathy via differential methylation (Li et al., 2014).
In addition, taking into account that this first intron harbors several ncRNA it can be hypothesized that the above mentioned SNP could alter some of them. MAPT-AS1 is an 840 bp long ncRNA transcribed from the anti-sense strand of the MAPT which has an inhibitory effect on MAPT promoter activity (Coupland et al., 2016). Interestingly, a significant decrease in MAPT-AS1 expression has been observed in PD (Coupland et al., 2016). Thus, it might be suggested that considering that the rs62056842 is close to the MAPT-AS1 promoter, its expression can be modulated by that particular SNP.

The study here commented on by Okbay et al. has only examined people of European ancestry and it is unclear whether the observed results apply to those with roots in other regions (Figure 1B). In fact, the 1000 genomes project (www.ncbi.nlm.nih.gov/variation/tools/1000genomes/) shows that, although rs62056842 presents an allele frequency of $T=$ 0.9147 and $G=0.0853$, being the allele $T$ the one associated with higher values of educational attainment in the meta-analysis carried out by the authors, that frequency shows great variation and, for example, it is not present in people from Africa or China where $T=1$. Overall, this study shows the importance of tau 
protein not only in learning, as different animal models have demonstrated, but also in educational attainment, being one of the genetic factors involved.

\section{AUTHOR CONTRIBUTIONS}

All authors listed, have made substantial, direct and intellectual contribution to the work, and approved it for publication.

\section{REFERENCES}

Andreadis, A. (2005). Tau gene alternative splicing: expression patterns, regulation and modulation of function in normal brain and neurodegenerative diseases. Biochim. Biophys. Acta 1739, 91-103. doi: 10.1016/j.bbadis.2004.0 8.010

Avila, J., Lucas, J. J., Perez, M., and Hernandez, F. (2004). Role of tau protein in both physiological and pathological conditions. Physiol. Rev. 84, 361-384. doi: 10.1152/physrev.00024.2003

Bradnam, K. R., and Korf, I. (2008). Longer first introns are a general property of eukaryotic gene structure. PLoS ONE 3:e3093. doi: 10.1371/journal.pone.0003093

Caffrey, T. M., and Wade-Martins, R. (2007). Functional MAPT haplotypes: bridging the gap between genotype and neuropathology. Neurobiol. Dis. 27, 1-10. doi: 10.1016/j.nbd.2007.04.006

Caillet-Boudin, M. L., Buée, L., Sergeant, N., and Lefebvre, B. (2015). Regulation of human MAPT gene expression. Mol. Neurodegener. 10:28. doi: 10.1186/s13024-015-0025-8

Chorev, M., and Carmel, L. (2012). The function of introns. Front. Genet. 3:55. doi: 10.3389/fgene.2012.00055

Coupland, K. G., Kim, W. S., Halliday, G. M., Hallupp, M., Dobson-Stone, C., and Kwok, J. B. (2016). Role of the long non-coding RNA MAPTAS1 in regulation of microtubule associated protein tau (MAPT) expression in Parkinson's disease. PLoS ONE 11:e0157924. doi: 10.1371/journal.pone.01 57924

Fernández-Nogales, M., Cabrera, J. R., Santos-Galindo, M., Hoozemans, J. J., Ferrer, I., Rozemuller, A. J., et al. (2014). Huntington's disease is a four-repeat tauopathy with tau nuclear rods. Nat. Med. 20, 881-885. doi: 10.1038/nm.3617

Gao, L., Tucker, K. L., and Andreadis, A. (2005). Transcriptional regulation of the mouse microtubule-associated protein tau. Biochim. Biophys. Acta 1681, 175-181. doi: 10.1016/j.bbaexp.2004.10.008

\section{ACKNOWLEDGMENTS}

This work was supported by grants from the following entities: Centro de Investigación en Red sobre Enfermedades Neurodegenerativas (CIBERNED, CB06/05/0066, Spain); the Spanish Ministerio de Economía y Competitividad (grants SAF 2015-66603-P, BFU2016-77885-P, and BUF201340664-P).

Huin, V., Deramecourt, V., Caparros-Lefebvre, D., Maurage, C. A., Duyckaerts, C., Kovari, E., et al. (2016). The MAPT gene is differentially methylated in the progressive supranuclear palsy brain. Mov. Disord. 31, 1883-1890. doi: $10.1002 / \mathrm{mds} .26820$

Iqbal, K., Liu, F., and Gong, C. X. (2016). Tau and neurodegenerative disease: the story so far. Nat. Rev. Neurol. 12, 15-27. doi: 10.1038/nrneurol.2015.225

Kwok, J. B., Teber, E. T., Loy, C., Hallupp, M., Nicholson, G., Mellick, G. D., et al. (2004). Tau haplotypes regulate transcription and are associated with Parkinson's disease. Ann. Neurol. 55, 329-334. doi: 10.1002/ana.10826

Li, Y., Chen, J. A., Sears, R. L., Gao, F., Klein, E. D., Karydas, A., et al. (2014). An epigenetic signature in peripheral blood associated with the haplotype on 17q21.31, a risk factor for neurodegenerative tauopathy. PLoS Genet. 10:e1004211. doi: 10.1371/journal.pgen.1004211

Myers, A. J., Pittman, A. M., Zhao, A. S., Rohrer, K., Kaleem, M., Marlowe, L., et al. (2007). The MAPT H1c risk haplotype is associated with increased expression of tau and especially of 4 repeat containing transcripts. Neurobiol. Dis. 25, 561-570. doi: 10.1016/j.nbd.2006.10.018

Okbay, A., Beauchamp, J. P., Fontana, M. A., Lee, J. J., Pers, T. H., Rietveld, C. A. et al. (2016). Genome-wide association study identifies 74 loci associated with educational attainment. Nature 533, 539-542. doi: 10.1038/nature17671

Conflict of Interest Statement: The authors declare that the research was conducted in the absence of any commercial or financial relationships that could be construed as a potential conflict of interest.

Copyright (c) 2017 Hernández and Ávila. This is an open-access article distributed under the terms of the Creative Commons Attribution License (CC BY). The use, distribution or reproduction in other forums is permitted, provided the original author(s) or licensor are credited and that the original publication in this journal is cited, in accordance with accepted academic practice. No use, distribution or reproduction is permitted which does not comply with these terms. 